\title{
Myocardial Extracellular Matrix Volume has independent prognostic value in patients with non-ischemic cardiomyopathy
}

\author{
Tomas S Vita*, Ethan Rowin, Siddique Abbasi, Xiaohu Li, Hoshang Farhad, Tomas G Neilan, Eri Watanabe, \\ François-Pierre Mongeon, Michael Givertz, Michael Steigner, Ron Blankstein, Marcelo DiCarli, \\ Michael Jerosch-Herold, Raymond Y Kwong
}

From 19th Annual SCMR Scientific Sessions

Los Angeles, CA, USA. 27-30 January 2016

\section{Background}

Left ventricular ejection fraction (LVEF) is the most validated independent prognostic factor for mortality, ventricular arrhythmias and heart failure (HF) in non-ischemic dilated cardiomyopathy without etiology. However, mild or moderate reduction LVEF yield limited predictive value.

The amount of fibrosis, detected by late gadolinium enhancement (LGE) on CMR, has also been validated as an independent predictor factor.

Conventional imaging techniques cannot robustly quantify the full spectrum of extracellular cardiac matrix volume (ECV) expansion. ECV expansion often may not be evident on LGE CMR or other modalities.

Quantifying ECV may ultimately provide independent prognostic value to improve care through targeted treatment.

The aim of this study was to determine the prognostic value of myocardial ECV expansion in patients with nonischemic cardiomyopathy.

\section{Methods}

Patients clinically referred for cardiac MRI evaluation between December 2008 and January 2014 with nonischemic cardiomyopathy with $\mathrm{LVEF}<60 \%$ were included in the study cohort. Primary endpoints included death and heart failure (HF) admissions. Elevated ECV was based on 2SD above normal volunteers sampling, at a cutoff of $34 \%$. T1 measurements were performed with a cine LookLocker sequence non-slice-selective inversion pulse, followed by segmented gradient-echo acquisition for 17 cardiac phases after inversion, spread over 2 cardiac cycles.

\section{Results}

Three hundred and fifty nine patients were included. The mean age was $50+/-16$ years; $60 \%$ were male; LV EF was $46+/-10 \%$; LV end diastole and end systole volume index $(\mathrm{mL} / \mathrm{m} 2)$ were $98+34$ and $56+34$, respectively. Mean LV mass was $60+/-21 \mathrm{~g}$. At a median follow-up of $2.5 \mathrm{yrs}$ (IQR $2.5 \mathrm{yrs}$ ), 22 deaths and $24 \mathrm{HF}$ admissions resulted. LGE was positive in $32 \%$ of patients. Depressed LV EF under $30 \%$ was present in $15 \%$ of the population. Twenty five percent of patients had an ECV mean over $34 \%$. Adjusted for LV EF $<30 \%$ and LGE presence, elevated ECV showed a significant association with death $(\mathrm{p}=0.009$; $\mathrm{HR}=3.83$; $\mathrm{CI}=1.39-10.51$ ).

\section{Conclusions}

1. ECV provides prognostic value for mortality of patients with non-ischemic CMP, incremental to LVEF and presence of myocardial scar by CMR.

2. Adjusted to LVEF of $<30 \%$ and LGE presence, ECV mean $>34 \%$ portends to a near 4 -fold increased risk for death.

Published: 27 January 2016

\section{doi:10.1186/1532-429X-18-S1-P226}

Cite this article as: Vita et al:: Myocardial Extracellular Matrix Volume has independent prognostic value in patients with non-ischemic cardiomyopathy. Journal of Cardiovascular Magnetic Resonance 2016 18(Suppl 1):P226.

Brigham and Womens Hospital, Boston, MA, USA 


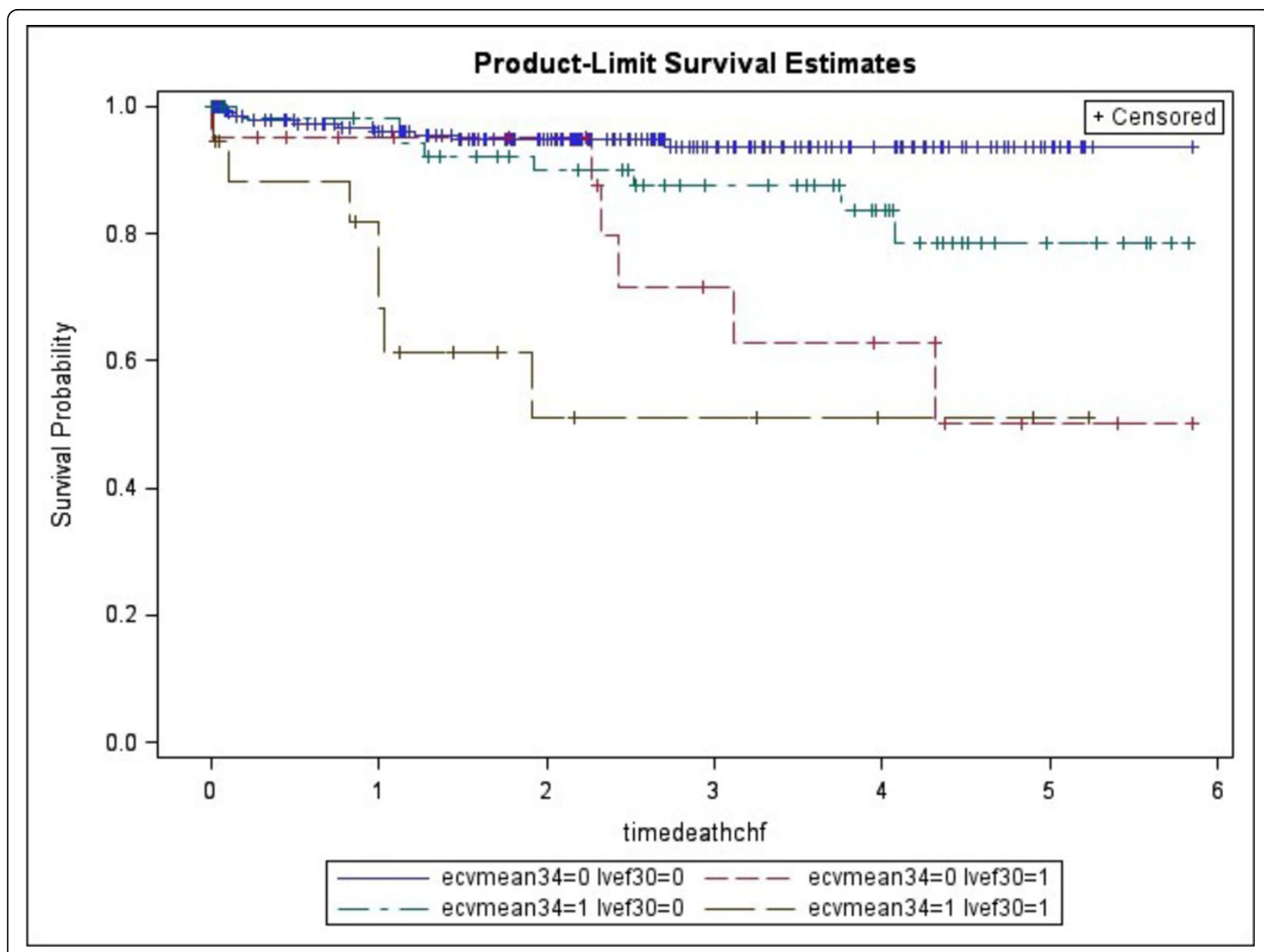

Figure 1 Plots of Kaplan-Meier product limit estimates of survival based on ECV and LVEF. 